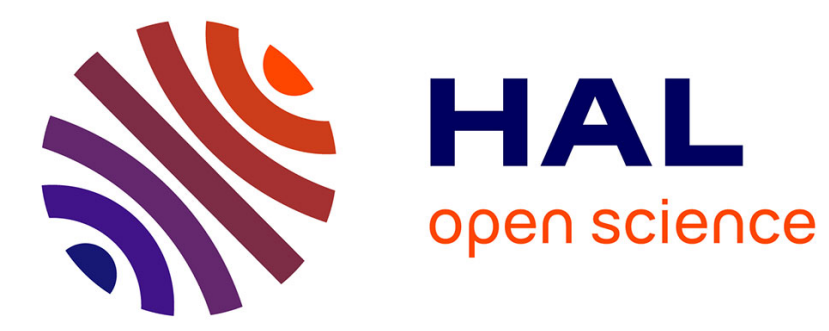

\title{
Daphné dans les textes français du Moyen Age: des amours réticentes
}

Christine Ferlampin-Acher

\section{To cite this version:}

Christine Ferlampin-Acher. Daphné dans les textes français du Moyen Age: des amours réticentes . Travaux de littérature, 2006, 58, p. 291-308. hal-01845689

\section{HAL Id: hal-01845689 \\ https://hal.science/hal-01845689}

Submitted on $20 \mathrm{Jul} 2018$

HAL is a multi-disciplinary open access archive for the deposit and dissemination of scientific research documents, whether they are published or not. The documents may come from teaching and research institutions in France or abroad, or from public or private research centers.
L'archive ouverte pluridisciplinaire HAL, est destinée au dépôt et à la diffusion de documents scientifiques de niveau recherche, publiés ou non, émanant des établissements d'enseignement et de recherche français ou étrangers, des laboratoires publics ou privés. 
Daphné dans les textes français du Moyen Age : des amours réticentes

C. Ferlampin-Acher (Rennes 2, CELAM-CETM)

L'aetas ovidiana du XIIe siècle nous a laissé des récits autonomes consacrés à Philomèle, Pyramus et Tisbé, Narcissus ${ }^{1}$. Pyrame et Narcisse, tout au long du Moyen Age, sont l'objet de réécritures, de gloses, d'allusions. Daphné semble avoir un destin plus modeste : elle n'inspire aucune traduction indépendante; son destin ne sert de trame à aucune transposition; rares sont les allusions à son histoire. Pourquoi étudier une figure si discrète ? Parce que les travaux consacrés aux métamorphoses les plus fameuses, Narcisse et Pirame, sont nombreux ${ }^{2}$. Parce que les silences (tels ceux de Daphné qui, muee -métamorphosée- est devenu muette ${ }^{3}$ ) sont parfois éloquents, et qu'il n'est pas vain de comprendre pourquoi une métamorphose a été négligée. Enfin parce que, comme l'a montré Yves Giraud ${ }^{4}$, le Moyen Age n'a pas complètement ignoré Daphné, comme en témoignent l'Ovide Moralisé, Froissart et Christine de Pizan.

\section{Daphné au Moyen Age : L'Ovide Moralisé, Froissart et Christine de Pizan}

La carrière de Daphné au Moyen Age commence bien. Comme le rappelle Yves Giraud, la nymphe a été l'une des rares figures païennes à échapper à l'hostilité des Pères de l'Eglise, grâce à sa chasteté 5 . Servius, Baudry de Bourgueil, Arnoul Greban, Jean de Garlande, les

\footnotetext{
${ }^{1}$ Voir l'éd. et la trad. anglaise de Raymond Cormier, Three Medieval Tales of Love, New York et Londres, Garland, 1986, ainsi que l'éd. et trad. française d'E. Baumgartner, Pyrame et Thisbé. Narcisse. Philomena, Paris, Folio, 2000.

${ }^{2}$ Voir par exemple E. Kölher, « Narcisse, la fontaine et Guillaume de Lorris », Journal des Savants, avr. juin 1963, p. 86-103 et Daniel Poirion, «Narcisse et Pygmalion dans Le roman de la Rose», dans Essays in Honor Francis Solano, Chapel Hill, 1970, p. 153-165. Pour Pirame et Tisbé, C. Segre «Piramo e Tisbé nei Lai di Maria di Francia », Studi in onore di V. Lugli et D. Valeri, Venice, 1961, vol. 2, p. 845-53 et Franz Schmitt-von Mühlenfels, Pyramus und Thisbe: Rezeptionstypen eines ovidisches Stoffes in Literatur, Kunst und Musik, Heidelberg, 1972.

${ }^{3}$ Ovide Moralisé, v. 3032 et 3060-1.

${ }^{4}$ La fable de Daphné. Essai sur un type de métamorphose végétale dans la littérature et dans les arts jusqu'à la fin du XVIIe siècle Genève, Droz, 1969 (p. 83-152 pour le Moyen Age).

${ }^{5}$ Op. cit., p. 87-ss.
} 
suivront. Daphné fait partie de la culture commune des clercs : Geoffroy de Vinsauf dans sa Poetria Nova tire du récit ovidien un exemple d'amplificatio.

Pourtant il faut attendre l'Ovide moralisé en vers (entre 1291 et 1328) ${ }^{6}$ pour que l'histoire de Daphné soit racontée dans un texte en langue vernaculaire : c'est presque 150 ans après Narcissus et Pyrame et Thisbé. L'Ovide moralisé reprend Ovide (nonobstant des ajouts assez nombreux) et fait suivre chaque "traduction adaptation" d'un commentaire plus ou moins nourri, conforme à la pratique médiévale de la glose ${ }^{7}$.

La "traduction" consacrée à Daphné suit d'assez près le texte latin. Les ajouts (les 116 hexamètres latins ont été amplifiés en 700 octosyllabes) sont significatifs. L'auteur insiste sur la métamorphose (v. 3032-ss), ce qui est surprenant : en général les reprises médiévales gomment les muances, choquantes pour des consciences chrétiennes (la métamorphose de Narcisse n'est pas évoquée dans les versions du XIIe siècle). De même l'enjeu étiologique (expliquer l'origine du laurier) est souligné (v. 2737-9), alors que dans d'autres métamorphoses de ce type, il est supprimé (c'est le cas pour Pirame) ${ }^{8}$. Le vœu de rester vierge et la course désespérée sont en revanche présentés plus longuement. Ces modifications valorisent la symbolique végétale et la chasteté. Le portrait de Daphné vue par Apollon ajoute une comparaison avec la rose et la fleur d'épine (v. 2890), qui, quoique topique, annonce la métamorphose végétale finale, et qui, comme le note M. Possamai, évoque la Passion du Christ ${ }^{9}$. Les identifications directes qui remplacent les périphrases latines facilitent la lecture et évitent de présenter peu chrétiennement Daphné comme la fille du fleuve Penée. Le gommage des allusions trop clairement antiques à Auguste ou au paganisme, et à l'inverse l'insertion d'éléments médiévaux, comme la liste des talents d'Apollon, reprenant les arts libéraux, vont de même dans le sens d'une adaptation au monde médiéval. Les deux ajouts concernant les flèches de Cupidon (v. 2750-3 et 2796-804) n'ont certainement pas un rôle explicatif, car le motif est alors bien connu: ils ont pour fonction de fédérer le lectorat autour

\footnotetext{
${ }^{6}$ Ed. C. de Boer, 5 vol., Amsterdam, 1915, 1920,1931, 1936, 1938 (Daphné : vol. I, 1. I, v. 2737-3412).

${ }^{7}$ Voir la thèse de M. Pérez-Possamai soutenue à Paris III en 2004 sous la direction de J. Dufournet, L'Ovide Moralisé: transtextualité et senefiance. Je remercie M. Pérez des références qu'elle m'a fournies.

${ }^{8}$ Voir mon article «Pyrame et Tisbé: le vert paradis des amours enfantines et la mort des amants», Lectures d'Ovide, mélanges Jean-Pierre Néraudau, textes réunis par E. Bury, Paris, Les Belles Lettres, 2003, p. 115-148.

${ }^{9} 7$ Thèse cit., p. 200.
} 
d'une culture commune. Les effets rhétoriques nombreux (allitérations, polyptotes, chiasmes) rappellent les romans du XIIe siècle : à ce titre l'Ovide moralisé est un texte très conservateur.

Ces différences s'expliquent par le point de vue de l'auteur : la métamorphose de Daphné doit être apprivoisée pour évoquer la Vierge Marie et la seule métamorphose acceptable pour une conscience chrétienne, l'Incarnation. Le récit ne cherche pas l'originalité : il passe par une culture commune, des procédés éprouvés et une clarté toute pédagogique, ce que confirment les gloses qui suivent.

Si toutes les métamorphoses n'ont pas le droit aux quatre gloses (naturelle, historique, morale et typologique), L'histoire de Daphné - c'est un signe de son importance - présente un modèle complet du travail interprétatif. La lecture naturelle y voit l'explication du fait que les lauriers aiment pousser au soleil (Phébus) et au bord de l'eau (le fleuve Penée) (v. 3065-ss); la lecture historique, évhémériste, y découvre l'histoire d'une femme, poursuivie par son amant, venant mourir près d'un laurier (v. 3102-ss) ; la lecture morale y voit un symbole de la chasteté associée à sapience et charité incarnées par Apollon. Enfin, la dernière sentance, typologique, montre dans Daphné la Vierge Marie; Cupidon est Dieu qui a sauvé les hommes (Apollon) en leur envoyant la bonne amour chrétienne, ses flèches représentent les comandement de la loy (v. 3215-ss).

Les interprétations typologiques proposées par l'Ovide moralisé eurent un succès très large. Pourtant, en ce qui concerne Daphné, ce n'est pas cette lecture que l'on retrouve dans $L^{\prime}$ Espinette amoureuse $(1370)^{10}$, dans Le Joli Buisson de Jonece $(1373)^{11}$ de Froissart ou dans l'Epitre Othea de Christine de Pizan (1401).

L'Espinette est une fiction «autobiographique », en vers, gonflée de développements mythologiques et de pièces lyriques. Le narrateur y évoque un amour de jeunesse. Entré dans un buisson où il a rencontré Vénus, il s'éprend d'une dame sur le point d'en épouser un autre. Malade, il compose une complainte en cinquante strophes (v. 1556-ss) $)^{12}$ centrée sur une juste

\footnotetext{
${ }^{10}$ Ed. A. Fourrier, Paris, Klincksieck , 1963.

${ }^{11}$ Ed. A. Fourrier, Genève, Droz, 1975.

${ }^{12}$ Sur cette complainte, voir B. Ribémont, «Froissart et le mythe de Daphné », Revue des langues romanes, 48, 1994, p. 189-199.
} 
comparison entre Daphné et ses propres amours. A la fin du texte, dans le " Réconfort de la dame", celle-ci reprend à son compte la figure de Daphné.

Froissart, grand relecteur et forgeur ${ }^{13}$ de mythes, propose une réécriture originale de l'histoire de Daphné centrée sur l'amour et la création. La figure masculine, Phebus, parfait amant courtois, est le centre de la fable, Daphné passant au second plan. Froissart montre Phébus, double du poète, frappé par un tison (v. 1565) lancé par Cupidon. Par un mouvement d'inversion, le dieu solaire reçoit la flamme. La métaphore du feu amoureux est valorisée : si Apollon est solaire, c'est parce qu'il aime Daphné. Réciproquement c'est un don de Phébus, après la métamorphose, qui rendra le laurier éternellement vers (v. 1752). La dimension étiologique ovidienne est réorientée : si l'amour et ses feux font de Phébus ce qu'il est, un être solaire, c'est Phébus qui crée le laurier. La parole poétique de l'amant a un pouvoir créateur. Cependant le regard porté sur la création n'est pas sans ambiguïté : lorsque le narrateur constate qu'il a moins de chance qu'Apollon qui pouvait adorer son laurier comme Pygmalion sa statue, l'amour devient à la fois fantasme et création artistique. Et lorsque le narrateur se repent d'avoir souhaité que sa dame soit changée en fourme villaine (v. 1816), n'est-ce pas qu'il s'inquiète des subterfuges de l'art et du fantasme?

Après ce détournement, relativement discret de la fable de Daphné, Froissart forge le mythe de Papirus et Idorée, dans lequel deux amants séparés peuvent se voir dans des miroirs magiques. Le mythe apparaît comme une consolation imaginaire, tout comme le miroir que le narrateur reçoit, dans lequel il voit son aimée lui offrir un poème, le "Réconfort de la dame". Ce texte reprend la figure de Daphné (v. 2861-ss), en insistant sur les souffrances de la nymphe et sur le laurier, symbole de ferme loyauté (v. 2872), d'amour fidèle. A nouveau, nous sommes loin de la chasteté de l'Ovide Moralisé. Mais la référence à Daphné est troublante. La dame commence en effet par mentionner Actéon qui tua par mégarde son amie cachée dans un buisson pour l'admirer (v. 2809-ss). Ce buisson reprend le buisson d'aubépines qui a servi d'intermédiaire entre le héros et son amie au début du récit. Si la réponse de la dame pouvait laisser espérer une issue heureuse, l'histoire d'Actéon, inaugurale, brise le charme. Ce Réconfort est donc fort

\footnotetext{
${ }^{13}$ D. Kelly, «Les inventions ovidiennes de Froissart : réflexions intertextuelles comme imagination », Literature, 1981, p. 82-92.
} 
incertain. Alors que le narrateur rêvait de créer son amie par les voies fantasmatiques du poème, à la fin du Réconfort, la dame lui dit qu'il sera toujours entés, escrips, figurés dans son cœur (v. 2882-2) : le poète devient lui-même représentation littéraire et rameau transplanté ; il est devenu Daphné. L'ambiguité de la fin du texte ${ }^{14}$ ainsi que le nom de la dame, Marguerite (ou Violette), qui désigne une fleur qui contrairement au laurier ne dure pas, renforcent l'idée d'un amour illusoire. C'est donc l'histoire d'une passion de jeunesse, médiatisée par les références littéraires (Cleomadès v. 705, Ovide v. 705) ${ }^{15}$. L'histoire de Daphné, déchristianisée, sans rapport avec la chasteté, ne doit rien à l'Ovide Moraliséet peu à l'auteur latin.

Trois ans plus tard, dans Le Joli Buisson de Jonece, le narrateur hésite à reprendre la plume et à aimer. Dans un songe Jeunesse cherche cependant à lui prouver l'éternelle jeunesse de son amie et s'appuie sur deux mythes inventés ${ }^{16}$ : celui d'Orphane, sœur de Daphné, aimée par le poète Architelés, et celui d'Ydropus et Neptiphoras (v. 2102-9 et v. 2015-92). Ces brèves forgeries reposent sur une onomastique qui en dénoncent l'inanité. En effet, pour le lecteur médiéval qui aime jouer sur les étymologies, Ydrophus évoque l'eau, Neptiphoras, Neptune. Ces deux personnages, d'emblée, ne sauraient illustrer les " feux de l'amour ". Quant à la Orphane, sa parenté avec Daphné ne la prédispose pas à la chaleur amoureuse et Architelés porte un nom qui, suggérant plus l'ancienneté que la jeunesse, est en décalage avec l'enjeu de la démonstration, l'éternelle jeunesse de l'amour? Par ailleurs le poète supplie Morphée de faire apparaître son amie Orphane, morte, dans ses rêves : tous deux s'aiment alors avec li acors de Dÿane. Cette caution amoureuse est étrange, d'autant que dans l'Espinette - les deux textes forment un diptyque - c'est Diane que Daphné invoque pour préserver sa chasteté. Même si Daphné, laurier éternellement jeune, peut être la sœur d'une Orphane qui ne vieillit pas, la forgerie de Jeunesse invite le lecteur à la méfiance.

\footnotetext{
${ }^{14}$ Voir W. Kibler, «Self-delusion in Froissart's Espinette amoureuse », Romania, 1976, p. 77-98 et «Le Joli buisson de Jonece: Froissart's midlife crisis », Froissart across the genres, éd. D. Maddox et S. Sturm-Maddox, Gainesville, University Press of Florida, 1998, p. 63-80.

${ }^{15}$ Voir B. Ribémont, « Froissart, le mythe et la marguerite », Revue des langues romanes, 94, 1990, p. 129-137 et «Froissart et le mythe de Daphné », Revue des langues romanes, 98, 1994, 189-199.

${ }^{16}$ Voir J. L. Picherit, «Le rôle des éléments mythologiques dans le Joli Buisson de Jonece », Neophilologus, 63, 1979, p. 498-508.
} 
Plus loin, le narrateur, caché dans un buisson, dit une ballade et tente de convaincre Désir que personne n'a été aussi enflammé par l'amour que lui. Désir le contredit, mentionnant, entre autres, Phébus amant de Daphné (v. 3154-ss) : alors même que le désir du narrateur semble sur le point de trouver un accomplissement, cet exemple est dysphorique, comme celui d'Actéon dans l'Espinette. Peu de temps après, avec le réveil du poète, le texte se termine par un lay de prière à la Vierge flour d'onnour tres souverainne/ En qui virginité maint (v. 5198-ss), contenant un résumé d'histoire sainte mentionnant le Buisson Ardent (v. 5373-ss). La virginité et les images florales suggèrent une substitution de la Vierge à Daphné, dans la continuité de l'Ovide Moralisé : le Buisson de Jeunesse à la lumière irisée et trompeuse est remplacé par le Buisson ardent qui ne brûle pas $^{17}$ dans lequel l'exégèse biblique voyait une préfiguration de Marie. La lecture personnelle et amoureuse du mythe de Daphné conduit finalement à sa conversion, dans l'esprit de l'Ovide Moralisé. Daphné détournée sert à illustrer l'échec d'un amour nourri d'images littéraires et illusoires dans l'Espinette; dans Le Joli Buisson de Jonece, elle permet de dénoncer une utilisation trompeuse des mythes et de proposer leur conversion, chrétienne et conservatrice.

Un autre exemple de reprise personnelle se lit dans l'Epitre Othea de Christine de Pizan ${ }^{18}$. Cet ouvrage traite cent exemples, selon un patron qui se répète : un quatrain en vers résume la fable, une glose en prose reprend le récit en ajoutant une ou deux explications et une citation de philosophe antique, puis une allegorie présente un enseignement chrétien et des citations des Pères de l'Eglise et de la Bible. Le modèle formel n'est pas éloigné de l'Ovide Moralisé et doit conforter le destinataire, le jeune Hector, dans ses bonnes dispositions.

Le quatrain en vers concernant Daphné (p. 323-4), ne retenant que la couronne de laurier, élude toute référence à l'amour, à la mort et la chasteté. Dans la glose en prose, la passion de Phébus, la virginité de l'héroïne et la thématique amoureuse sont rapidement évoquées et mises de côté. La course de Phébus symbolise d'abord la quête de l'honneur, puis la perseverence du chrétien qui vise le Paradis et ses joies infinies. L'inversion est nette : si dans l'Ovide Moralisé Daphné incarne la valeur célébrée, la chasteté, elle passe ici au second plan, sa métamorphose

\footnotetext{
${ }^{17}$ On retrouve les deux polarisations de la lumière médiévale. Cf. A. Planche, «Du joli Buisson de Jonece au Buisson Ardent : le lay de Notre Dame dans le Dit de Froissart », La prière au Moyen Age, Senefiance, 1980.

${ }^{18}$ Ed. G. Parussa, Genève, Droz, 1999.
} 
n'intéresse pas : le modèle est Phébus et sa persévérance. Dans ce miroir au prince, Daphné reste dans l'ombre.

Les allégorisations de Froissart et Christine de Pizan sont toutes deux marquées par l'Ovide moralisé. Si Froissart s'éloigne sur le plan formel de cette source, il en retrouve in fine la lecture chrétienne et mariale. A l'inverse Christine, dans un texte formellement proche de l'Ovide moralisé, réussit à ne mentionner ni la chasteté ni la Vierge Marie.

\section{Des réticences}

Malgré ces trois exemples, Daphné est peu présente. Pourquoi ?

Peut-être à cause de son nom. La forme médiévale attestée dans l'Ovide Moralisé ou chez Christine est Dané, Dane, Dauné qui pouvait se confondre avec Dané, forme de Danaé. Une confusion similaire est attestée en latin ${ }^{19}$. Cependant la rareté des allusions à Danaé et le fait que dans l'Ovide Moralisé coexistent dans le livre I Dané / Daphné (v.2740) et Dané / Danaé (v. 5493 ) invalident cet argument. D'autre part, certaines graphies conservent le $p$ (Dafpes chez Geoffroy de Vinsauf, Dampné chez Christine de Pizan). Le rapprochement dampné / Dané, entre la damnation et la chaste nymphe, était peut-être problématique, même s'il est exploité par l'Ovide Moralisé (1. I, v. 3172-3) qui juxtapose les deux termes pour mieux les opposer : ce jeu était possible dans un texte qui glose clairement la sainteté de la figure, mais dans un texte narratif non didactique, sans glose développée, l'ambiguité risquait d'être gênante, d'autant qu'au Moyen Age par le non conuist an l'ome ${ }^{20}$. Une héroïne signalée d'emblée par sa «damnation» pouvait poser un problème, mais l'exemple du Narcissus sur lequel je reviendrai plus loin invalide cette idée. D'ailleurs ce nom offre d'autres possibilités poétiques très intéressantes : chez Froissart, la métrique nous invite à lire Dane plutôt que Dané: cette forme se rapproche à la fois de Diane et de dame qui désigne à la fois la femme aimée et Nostre Dame, la Vierge Marie. Ce

\footnotetext{
${ }^{19}$ Voir E. Faral, Les arts poétiques du XIIe et du XIIIe siècle, Paris, 1962, p. 216, v. 620 et la note. On remarque que la confusion a pu être facilitée par la proximité entre aurum et laurum.

${ }^{20}$ Chrétien de Troyes, Le Conte du Graal, éd. F. Lecoy, Paris, Champion, 1973, v. 560 «On connaît l’homme à son nom ».
} 
nom, pouvant sans peine servir des lectures amoureuse et chrétienne n'explique donc pas le désamour des auteurs médiévaux pour la figure.

Une deuxième hypothèse peut être avancée. Les auteurs sont réticents face à la métamorphose végétale (celle de Narcisse est en général gommée). Plus encore que la métamorphose animale qui peut être glosée moralement ${ }^{21}$, la métamorphose florale est difficilement acceptable, sauf dans un texte comme l'Ovide moralisé dont l'enjeu justement est de tout moraliser. Cependant, à défaut de pouvoir devenir un véritable personnage dont l'histoire serait menée à terme, jusqu'à la muance, Daphné aurait pu servir de comparaison, comme Pirame ou Narcisse, qui souvent ne sont qu'un nom, parmi plusieurs, illustrant l'amour, son idéal et ses dangers. Telle héroïne repoussant une passion pour mieux en exalter la courtoisie et la mettre à l'épreuve aurait pu être comparée à Daphné. Ce n'est pas le cas : trop liée à la célébration de la virginité, Daphné était peu compatible avec les enjeux romanesques. L'absence de Daphné dans le Roman de la Rose (ce texte exclut du paradis ceux qui ont commis le péché de virginité v. 19612) a d'ailleurs certainement nui à sa postérité littéraire.

La concurrence avec d'autres images a pu aussi jouer contre Daphné. L’imagination de l'homme médiéval est visuelle. Or la métamorphose de Daphné en laurier suggère une étape intermédiaire, où l'héroïne est mi-femme, mi-arbre. C'est ainsi que les rares miniaturistes à s'être risqués à illustrer la scène la montrent ${ }^{22}$. Or les représentations qui mêlent l'arbre et l'humain sont nombreuses au Moyen Age ${ }^{23}$ : arbre de Jessé, songe d'Astyage, Christ en Croix, arbres de type généalogique, serpent de la tentation dans l'arbre du péché originel. Restait-il de la place pour une Daphné mi-femme mi-arbre?

\footnotetext{
${ }^{21}$ Sur ce point l'influence du livre IV de la Consolation de Philosophie de Boèce a joué un rôle essentiel. Voir A. Pairet, Les mutacions des fables. Figures de la métmorphose dans la littérature française du Moyen Age, Paris, Champion, 2002, p. 41. Sur la préférence accordée aux métamorphoses animales lues comme métaphores morales, ibid., p. 59-ss. Froisart, dans le Voyage en Béarn, signale: Nous trouvons en l'escripture que anciennement les dieux et les deesses a leur plaisance muoient les hommes en bestes et en oyseaux, et aussi faisoient les femmes (éd. S. Luce, Chroniques, vol. XII, livre III, p. 84-85). Il élude les métamorphoses végétales et enchaine sur Actéon.

${ }^{22}$ Voir P Y. Giraud, op. cit., planche 7.

${ }^{23}$ Voir J. Baltrusaitis, Le Moyen Age fantastique, Flammarion, 1981, p. 114ss.
} 
L'Arbre de Jessé, inspiré de la prophétie d'Isaie (XI, 1) ${ }^{24}$, annonce que le patriarche Jessé donnera naissance à une tige et à une fleur, qui sont interprétées au Moyen Age comme étant le Christ et la Vierge. Dans les représentations iconographiques, l'arbre sort de la bouche (assez rarement), du ventre, du cœur ou du dos de Jessé. Tantôt l'arbre porte des têtes couronnées (BnF. fr. 10), des troncs couronnés (BnF. fr.159), la généalogie de la Vierge (BnF. fr. 157): la Vierge peut se trouver en haut ou au milieu, avec ou sans Jésus. L'arbre de Jessé, associé à la généalogie de la Vierge et la venue du Christ, n'était pas incompatible avec la lecture chrétienne de Daphné de l'Ovide moralité. La concurrence entre les images n'était pas a priori problématique.

Il en va de même pour le songe d'Astyage ${ }^{25}$. Tantôt le rêveur est allongé, et regarde une vigne géante qui sort d'une femme, debout près du lit $(\mathrm{BnF}$. fr. $188, \mathrm{f} .7 \mathrm{v})$, tantôt on voit Mandane allongée avec une vigne qui sort de son ventre (BnF. fr. 50, f. 91v). Ce songe est parfois mis en parallèle avec celui de Jessé et avec une thématique mariale : dans les bois gravés du Speculum Humanae Salvationis, un diptyque présente à gauche l'annonce de la naissance de Marie et à droite Astyage ; un autre montre à gauche la Nativité et à droite Jessé ${ }^{26}$. La lecture mariale qui est faite du songe d'Astyage dans ce contexte le rend compatible avec Daphné.

La scène du péché originel, qui souvent montre dans un arbre une créature hybride à tête ou à tronc de femme, représentant le démon ${ }^{27}$, greffe de même un corps humain et des éléments végétaux. Elle a un sémantisme qu'on aurait tort de croire incompatible avec Daphné, puisque Eve est rachetée par Marie. Les Crucifixions présentant une croix égotée, c'est-à-dire faite avec

\footnotetext{
${ }^{24}$ Voir A. Strubel, «Allegoria in factis et allegoria in verbis », dans Poétique, 1975, n²3, p. 342-357 où l'on trouvera des commentaires concernant l'arbre de Jessé. G. Duby consacre une page et une illustration à ce motif : Le Moyen Age, 1140-1280, Skira, Genève, 1984, p. 30-34.

${ }^{25}$ Selon Hérodote, Astyage rêva que du ventre de sa fille Mandane sortait un torrent qui couvrait l'Asie entière: pour ses mages, ce rêve annonce la future domination des Mèdes sur l'Asie entière. Inquiet pour son trône, Astyage choisit pour époux à sa fille un Perse de condition inférieure. Un second songe lui fait voir une vigne sortant du ventre de sa fille et couvrant l'Asie, ce qui, pour les mages, confirmait l'hégémonie mède. Ce récit est très connu au Moyen Age. On le trouve par exemple dans le Speculum Historiale de Vincent de Beauvais.

${ }^{26}$ Reproduit dans The Mirror of Salvation, an édition of British Library Blockbook G. 11784, trad. A. Labriola et J. Smeltz, Duquesne University Press, 2002, p. 22 et 24.

${ }^{27}$ Voir par exemple la Bible Historiale de Guiard des Moulins, BnF. fr. 3, f. 8v et BnF. fr. 159 f. 8.
} 
des troncs d'arbres juste ébranchés, où le Christ fait corps avec l'arbre, tout comme le rapport entre l'arbre de vie et l'arbre de la Croix, renforçant l'analogie entre l'arbre et la croix ${ }^{28}$, invitent d'ailleurs à mettre en rapport de l'arbre et le rachat des hommes par le Christ, ce qui va dans le même sens que la lecture chrétienne de Daphné et du laurier.

Ainsi on aurait pu penser que l'iconographie de l'arbre humain était saturée : en fait, le Moyen Age ne craint pas les polysémies, et pousse, comme l'Ovide Moralisé nous le montre, fort loin l'acceptation de lectures parallèles et pour nous hétérogènes, voire divergentes. L'argument selon lequel le mythe de Daphné se serait peu développé à cause de la concurrence d'autres images mêlant l'arbre et le corps humain, n'est donc pas pertinent.

Une dernière hypothèse peut être présentée : Daphné aurait été concurrencée par Narcisse, qui a eu un succès durable au Moyen Age. Les parallèles entre les deux histoires sont nombreux : Narcisse reste sourd aux supplications d'Echo, comme Daphné à celles de Jupiter, et comme Daphné, il se métamorphose en végétal. Pourtant Froissart, grand amateur et forgeur de mythes, a été simultanément fasciné par Narcisse et Daphné dans l'Espinette amoureuse (voire dans Le Joli Buisson de Jonece $)^{29}$ et Christine de Pizan fait se succéder les histoires d'Echo et Daphné dans l'Epitre Othea. Narcisse et Daphné pouvaient fort bien se développer parallèlement. Les gloses de l'Ovide Moralisé les distinguent d'ailleurs : Daphné, sa constance et le feuillage persistant du laurier s'opposent à Narcisse qui représente l'orgueil fasciné par de vaines illusions et qui se transforme en une floerete vite fanée (III, 1847-ss ${ }^{30}$ ).

Ainsi aucun de ces arguments n'explique la discrétion de la nymphe dans la littérature médiévale. Plus encore chacun d'eux laisse supposer au contraire que le mythe de Daphné pouvait se greffer, dès lors qu'il était christianisé dans l'Ovide Moralisé, sur des thèmes, des motifs, des représentations, en vogue : ce qui m'incite à penser que si Daphné est rarement mentionnée explicitement, en revanche son histoire a travaillé en profondeur les imaginaires.

\footnotetext{
${ }^{28}$ Voir G. Dufour-Kowalska, L'arbre de vie et la croix: essai sur l'imagination visionnaire, Genève, éd. du Tricorne, 1985.

${ }^{29}$ Sur le miroir et Narcisse chez Froissart, voir F. Pomel, «Du miroir-mire de l'amant à l'écrivain miroitier. Les trompe-l'œil de l'imagination dans L'Espinette amoureuse et Le Joli Buisson de Jonec de Froissart », dans Miroirs et jeux de miroirs dans la littérature médiévale, éd. F. Pomel, Presses Universitaires de Rennes, 2003, p. 288-302 .

${ }^{30}$ Même chose dans l'Epitre Othea, exemple 16.
} 
Trois exemples, éloignés dans le temps et la forme ( Narcissus, Cristal en Clarie, et Perceforest), montrent que l'histoire de Daphné est effectivement un palimpseste aussi persistant que le laurier.

\section{Daphné retrouvée}

Le lai de Narcissus $^{31}$, en plein aetas ovidiana du XIIe siècle, est le résultat d'une greffe entre Narcisse et Daphné dans laquelle Echo disparait, remplacée par un personnage nommé Dané. Si A. Pairet ${ }^{32}$ assimile Dané et Danaé, et si M. Pelan et N. Spece, dans leur glossaire, rendent Dané par Daphné, M. Thiry dans un article de 1978 a conclu non seulement que le nom de Dané correspond à Daphné, mais aussi que l'histoire de cette dernière est une source du Narcissus $^{33}$ : l'auteur a interverti les rôles masculins et féminins, Narcisse étant poursuivi amoureusement comme l'est Daphné, jusqu'à la métamorphose finale et florale.

Aux arguments présentés par M. Thiry, on peut en ajouter d'autres. La paronomase Dané/ dampné, qui aurait pu nuire, comme je l'ai vu plus haut, à la nymphe, a en fait servi ici son réemploi. En effet, dans le Narcissus, Dané ne se métamorphose pas : elle se suicide. Cette fin, choquante pour un lecteur chrétien du XIIe siècle, entrait en résonance avec le nom propre qui pouvait prendre la forme Dampné ${ }^{34}$. Par ailleurs l'importance de l'eau, paternelle pour Daphné, nourricière pour le laurier, source pour Narcisse, favorise le rapprochement. L'inversion des rôles masculin et féminin est d'autre part à mettre en relation avec le personnage de Tirésias, homme et femme, qui certes a disparu dans le Narcissus mais qui chez Ovide introduit l'histoire de Narcisse (III, v. 316-ss).

De plus, si le Narcisse médiéval ressemble à la Daphné antique, Dané a des points communs autres que le nom avec la nymphe antique : tout comme Daphné échappe au regard de Phébus en se faisant arbre, Dané se cache dans un arbre pour admirer Narcisse (v. 429); Dané

\footnotetext{
${ }^{31}$ Narcisse, éd. M. Thiry-Stassin et M. Tyssens, Paris, Les Belles Lettres, 1976 ; Narcisus, éd. M. M. Pelan et N. Spence, Les Belles Lettres, 1964 . Voir l'éd. et la trad. de Raymond Cormier, cit. n. 1. Les références données sont celles de l'éd. de 1976.

${ }^{32}$ Op. cit., p. 48-ss.

33 «Une autre source ovidienne du Narcisse », Le Moyen Age, 84, 1978, p. 211-226.

${ }^{34}$ Sur le suicide de Dané, voir M. N. Lefay-Toury, La tentation du suicide dans le roman français du XIIe siècle, Paris, Champion, 1979, p. 57-ss.
} 
adresse une prière aux dieux du ciel et de la terre (v. 608-ss), tandis que Daphné invoque la terre, à laquelle elle demande de l'engloutir (v. 3021). Narcisse prend Dané pour une déesse ou une fée (v. 450), alors que le récit la présente comme une simple demoiselle : en dépit du caractère topique de cette confusion, n'est-ce pas, subtilement, un rappel de la nymphe antique, les nymphes, fées et déesses étant souvent confondues au Moyen Age ? Par ailleurs Phébus et Dané échangent aussi des traits : tous deux subissent la blessure d'amour; tous deux vantent leur lignage. Ainsi non seulement il y a eu substitution de nom entre Daphné et Dané, non seulement il y a inversion des rôles masculins et féminins entre Daphné et Narcisse, mais plus généralement Daphné, Phébus, Narcisse, Dané, échangent leurs traits. Quand Narcissus voit Dané, il pense que c'est une déesse ou une fée, puis s'admirant à la surface de l'eau, il s'adresse à son reflet, au féminin, en suggérant que c'est ninphe u fee (v. 677), avant de voir, juste avant de mourir, non pas son propre reflet, mais Dané (v. 965): entre Dané et Daphné, puis entre Daphné et Narcisse, enfin entre Narcisse et Dané, la spécularité amoureuse est en fait ternaire, comme si le fantasme de la nymphe s'interposait entre le désir du héros et la réalité. Certes au XIIe siècle comme dans l'Antiquité Narcisse meurt d'un désir égocentrique, mais dans le texte médiéval, son désir le porte surtout à voir des nymphes, des Daphné, là où il n'y a que des Dané (des mortelles) ou luimême. Le fantasme, qui est à la fois lui et l'autre, l'empêche d'aimer dans la réciprocité. Ces jeux de miroirs, qui ne sont pas surprenants dans un texte à la thématique spéculaire, montrent qu'il n'y a donc pas seulement une substitution de nom ou de sexe : la nymphe antique est à l'horizon du texte un fantasme à la fois amoureux et littéraire ; elle est le manque sur lequel est construit le récit.

Cette greffe entre Daphné et Narcisse est, à mon avis, particulièrement motivée et réussie. Elle n'est d'ailleurs peut-être pas restée stérile. Je me demande en effet si Cristal et Clarie (roman en vers du XIIIe siècle ${ }^{35}$ ) ne rapproche pas Narcisse et Daphné. L'auteur connaissait bien Narcissus et n'a pas hésité à «farcir» son texte de citations du lai ovidien. Au cours de ses aventures, le héros arrive près d'une fontaine (v. 5840-ss). Dans un arbre se trouve une demoiselle qui lui explique qu'il mourra s'il boit car un luiton démoniaque se trouve dans l'eau. Elle raconte qu'elle vit dans cet arbre et dans la chapelle voisine depuis douze ans pour échapper

${ }^{35}$ Cristal et Clarie, éd. H. Breuer, Dresde, 1915. 
à ce diable concupiscent. Ce triste sort s'explique par la vengeance d'une fée, repoussée par le père de la demoiselle. Les points communs entre Narcisse et Cristal sont nombreux : il serait mortel pour Cristal de se mirer à la fontaine ; Cristal repousse souvent, comme Narcisse, l'amour des pucelles qu'il rencontre, ce qui donne l'occasion à l'auteur d'insérer des vers du Narcissus (v. 1351-70 ; v. 2437-69). L'aventure entretient aussi des rapports avec l'histoire de Daphné : la demoiselle, près de l'eau, se réfugie dans un arbre pour échapper au désir d'un luiton (ce qui pourrait se lire comme une rationalisation de la métamorphose); le luiton est une créature païenne, comme Apollon; son père a refusé l'amour d'une fée, tout comme Daphné a rejeté Apollon. Le nom de la demoiselle, Lynarde, évoque la narde, une graminée qui pousse dans les eaux humides. Si les Marguerite et les Florence sont fréquentes parmi les héroïnes médiévales, cette Lynarde atypique évoque le laurier de Daphné, à cause de ses feuilles raides et de son goût pour les zones aquatiques ${ }^{36}$. Cette Lynarde a d'ailleurs une cousine, appelée précisément Narde. Cristal et Clarie, roman très subtil, a certainement su retrouver Daphné dans le Narcissus et a à son tour, exploité le rapprochement.

Ainsi la concurrence entre Daphné et Narcisse a permis une greffe, le Narcissus, dont le succès sera durable. Cependant, malgré Cristal et Clarie, les fruits seront peu nombreux. Il n'est pas impossible d'ailleurs que la vogue du Narcissus ait finalement bloqué le réemploi du nom de Dané/ Daphné.

Outre le nom, un problème soulevé par la reprise du mythe de Daphné était, nous l'avons vu, la concurrence d'images jouant sur l'hybridation entre l'homme et le végétal. Or un épisode du roman en prose Perceforest témoigne d'une résurgence du mythe de Daphné au XIVe siècle que l'on peut justement décrypter grâce à ces images. Notons d'emblée que la culture encyclopédique de l'auteur de Perceforest garantit qu'il connaissait les Métamorphoses, en particulier Narcisse : il donne à l'un de ses personnages principaux le nom de Liriopé et multiplie les épisodes à jeux de miroirs.

Dans ce roman, un système de trois songes reprend la lecture chrétienne de Daphné: dans le livre II, le rêve de Gadifer (II, I, p. 85-86) et celui de Perceforest (II, I, p. 232-ss) et dans le

\footnotetext{
${ }^{36}$ Voir mon article «Cristal et Clarie et Perceforest: un problème de taille, du petit chevalier au Bossu de Suave », à paraître dans les Mélanges F. Dubost, Paris, Champion.
} 
livre V celui de Galafur (f. 272) ${ }^{37}$. La cohérence de cet ensemble, implicite, repose sur la métamorphose en arbre et l'interprétation mariale de Daphné. Comme je l'ai montré ailleurs ${ }^{38}$, le premier rêve montre un homme qui prophétise dans un laurier. Le second est aussi prophétique : une forêt qui dépérit symbolise la décadence du royaume mal gouverné. Dans le troisième, la croissance d'une forêt dont les arbres se transforment en hommes illustre la restauration de la Bretagne, puis le rêveur voit l'intérieur de son corps, les ramifications de ses veines et trois gouttes de son sang qui deviennent trois enfants, tandis que la dernier partie de ce songe aboutit au Chevalier Vierge, qui à la fin de l'épisode recevra l'écu marqué d'une croix christique et apprendra à combattre au nom du Fils de la Vierge. Sans reprendre ce que j'ai déjà tenté de montrer ailleurs, la métamorphose en arbre, la mention du laurier, l'importance de la chasteté, la dimension généalogique et la référence au Christ et à la Vierge coïncident à la fois avec les prophéties de Merlin, telles qu'elles figurent dans 1'Historia Regum Britanniae 39 et avec l'histoire de Daphné et sa lecture chrétienne. Cependant lorsque la Reine Fée est interrogée sur la métamorphose en arbre, elle se contente de dire que la volonté de repeupler le pays est telle qu'il n'eust sur terre arbres ne pierre qui ne se muast en figure d'homme ou de femme. Cette explication, cavalière, juxtapose des métamorphoses en pierre et en arbre. Or la métamorphose en pierre n'a a priori rien à voir avec le songe et on n'en trouve aucune trace ailleurs dans le texte. Pourquoi cette allusion? Dans un précédent travail, j'avais pensé qu'il pouvait s'agir d'une allusion à Deucalion, dont l'histoire est voisine de celle de Daphné chez Ovide et dans l'Ovide moralisé, ou aux Propétides. Finalement, je ferai aujourd'hui une autre proposition : les os d'Echo chez Ovide étant transformés en pierres (Ovide Moralisé 1. III, v. 1457), la réponse de la Reine Fée rapprocherait Echo et Daphné, comme l'a déjà fait le Narcissus. Daphné serait un

\footnotetext{
${ }^{37}$ Ce travail s'appuie sur les éditions suivantes: Perceforest (le roman de), deuxième partie, éd. G. Roussineau, Genève, T. L. F., vol. I, 1999 et vol. II, 2001. Le livre V est inédit : les références renvoient au manuscrit BnF fr. 348.

${ }^{38}$ Je renvoie à mon article à paraître dans les Actes du XIIe Colloque international sur le moyen français, McGill, 2004, «Fleurs de rhétorique, Buissons Ardents et Arbres de Jessé : autour de quelques comparaisons, métaphores et paraboles dans Perceforest $»$.

${ }^{39}$ Ed. N. Wright, Cambridge, 1984, p. 78-ss.
} 
palimpseste très effacé, avec en surimpression l'arbre généalogique de Jessé, qui conduit à la Vierge et renvoie à l'Immaculée Conception.

Daphné est discrètement présente, à l'ombre de Narcisse ou de la Vierge. A travers elle le désir se trouve questionné et une sexualité chaste se rêve, comme celle que l'on prêtait alors aux plantes. Peu présente, fantasme, image, Daphné doit être poursuivie avec assiduité, et elle nous échappe finalement : nous en sommes réduits à des suppositions. Mais finalement, si Narcissus a rejeté Echo et ses répétitions mortelles et a préféré Daphné, c'est qu'au soliloque mortifère le poète ne peut que préférer la métamorphose de l'humain en foeilles ${ }^{40}$, qu'elles soient de laurier ou de vélin.

\footnotetext{
${ }^{40}$ La polysémie du terme est attestée au Moyen Age.
} 